\title{
EXISTENCE OF SOLUTIONS OF SECOND ORDER NEUTRAL FUNCTIONAL DIFFERENTIAL EQUATIONS
}

\author{
K. BALACHANDRAN AND S. MARSHAL ANTHONI
}

\begin{abstract}
Albstract. Sufficient conditions for existence of mild solutions for second order neutral functional differential equations are established by using the theory of strongly continuous cosine families and the Schaefer theorem.
\end{abstract}

\section{Introduction}

In many cases it is advantageous to treat the second order abstract differential equations directly rather than to convert them to first order equations. A useful machinery for the study of abstract second order differential equations is the theory of strongly continuous cosine families. We will make use of some of the basic ideas from cosine family theory $[8,9]$. Motivation for second order systems can be found in $[1,3,4]$. Recently, Ntouyas [5] and Ntouyas and Tsamatos [6] established the existence of solutions for semilinear second order delay differential equations using the Schaefer theorem. The purpose of this paper is to study the existence of mild solutions for second order neutral functional differential equations using the Schaefer theorem.

\section{Premilinaries}

Consider the second order neutral functional differential equation of the form

$$
\begin{aligned}
\frac{d}{d t}\left[x^{\prime}(t)-g\left(t, x_{t}\right)\right] & =A x(t)+f\left(t, x_{t}, x^{\prime}(t)\right), \quad t \in J=(0, T], \\
x_{0} & =\phi, \quad x^{\prime}(0)=y_{0},
\end{aligned}
$$

where the state $x(\cdot)$ takes values in a Banach space $X, y_{0} \in X, A$ is the infinitesimal generator of the strongly continuous cosine family $C(t), t \in R$, of bounded linear operators in $X, f: J \times C \times X \rightarrow X$ and $g: J \times C \rightarrow X$ are given functions and $\phi \in C$.

Here $C=C([-r, 0], X)$ is the Banach space of all continuous functions $\phi:[-r, 0] \rightarrow X$ endowed with the sup-norm.

$$
\|\phi\|=\sup \{|\phi(\theta)|:-r \leq \theta \leq 0\} .
$$

Received December 7, 1998.

1991 Mathematics Subject Classification. 34K30, 47H15.

Key words and phrases. Neutral functional differential equation, Cosine operators, Schaefer theorem. 
Also for $x \in C([-r, T], X)$ we have $x_{t} \in C$ for $t \in J, x_{t}(\theta)=x(t+\theta)$ for $\theta \in[-r, 0]$.

Definition 1. [8] A one parameter family $C(t), t \in R$, of bounded linear operators in the Banach space $X$ is called a strongly continuous cosine family iff

(i) $C(s+t)+C(s-t)=2 C(s) C(t)$ for all $s, t \in R$;

(ii) $C(0)=I$;

(iii) $C(t) x$ is continuous in $t$ on $R$ for each fixed $x \in X$.

Define the associated sine family $S(t), t \in R$, by

$$
S(t) x=\int_{0}^{t} C(s) x d s, \quad x \in X, t \in R .
$$

Assume the following conditions on $A$.

$\left(H_{1}\right) A$ is the infinitesimal generator of a strongly continuous cosine family $C(t), t \in R$, of bounded linear operators from $X$ into itself and the adjoint operator $A^{*}$ is densely defined i.e. $\overline{D\left(A^{*}\right)}=X^{*}$ (See [2].)

The infinitesimal generator of a strongly continuous cosine family $C(t), t \in R$, is the operator $A: X \rightarrow X$ defined by

$$
A x=\left.\frac{d^{2}}{d t^{2}} C(t) x\right|_{t=0}, \quad x \in D(A),
$$

where $D(A)=\{x \in X: C(t) x$ is twice continuously differentiable in $t\}$.

Define $E=\{x \in X: C(t) x$ is once continuously differentiable in $t\}$.

To establish our main theorem we need the following lemmas.

Lemma 2.[8] Let $\left(H_{1}\right)$ hold. Then

(i) there exist constants $M \geq 1$ and $\omega \geq 0$ such that $|C(t)| \leq M e^{\omega|t|}$ and $\mid S(t)-$ $S\left(t^{*}\right)|\leq M| \int_{t}^{t^{*}} e^{\omega|s|} d s \mid$ for $t, t^{*} \in R$

(ii) $S(t) X \subset E$ and $S(t) E \subset D(A)$ for $t \in R$;

(iii) $\frac{d}{d t} C(t) x=A S(t) x$ for $x \in E$ and $t \in R$;

(iv) $\frac{d^{2}}{d t^{2}} C(t) x=A C(t) x$ for $x \in D(A)$ and $t \in R$.

Lemma 3.[8] Let $\left(H_{1}\right)$ hold, let $v: R \rightarrow X$ such that $v$ is continuously differentiable and let $q(t)=\int_{0}^{t} S(t-s) v(s) d s$. Then

$q$ is twice continuously differentiable and for $t \in R, q(t) \in D(A)$,

$$
q^{\prime}(t)=\int_{0}^{t} C(t-s) v(s) d s, \text { and } q^{\prime \prime}(t)=A_{q}(t)+v(t) .
$$

Lemma 4.(Schaefer Theorem [7]) Let $S$ be a convex subset of a normed linear space $Y$ and assume $0 \in S$. Let $F: S \rightarrow S$ be a completely continuous operator, and let

$$
\xi(F)=\{x \in S: x=\lambda F x \text { for some } 0<\lambda<1\} .
$$


Then either $\xi(F)$ is unbounded or $F$ has a fixed point.

Definition. A continuous function $x:[-r, T] \rightarrow X, T>0$, is called a mild solution of the problem (1) if $x_{0}=\phi$, and if it satisfies the integral equation

$$
\begin{aligned}
x(t)= & C(t) \phi(0)+S(t)\left[y_{0}-g(0, \phi)\right]+\int_{0}^{t} C(t-s) g\left(s, x_{s}\right) d s \\
& +\int_{0}^{t} S(t-s) f\left(s, x_{s}, x^{\prime}(s)\right) d s, \quad t \in J .
\end{aligned}
$$

We make the following assumptions:

$\left(H_{2}\right) C(t), t>0$ is compact.

$\left(H_{3}\right)$ The function. $g$ is completely continuous and such that the operator

$$
G: C([-r, 0], X) \rightarrow C([0, T], X)
$$

defined by $(G \phi)(t)=g(t, \phi)$ is compact.

$\left(H_{4}\right)$ There exist constants $c_{1}$ and $c_{2}$ such that

$$
|g(t, \phi)| \leq c_{1}\|\phi\|+c_{2}, \quad t \in J, \quad \phi \in C .
$$

$\left(H_{5}\right)$ The function $f(t, .,):. C \rightarrow X$ is continuous for each $t \in J$.

$\left(H_{6}\right)$ The function $f(., x, y): J \rightarrow X$ is strongly measurable for each $x \in C$ and $y \in X$.

$\left(H_{7}\right)$ For every positive constant $k$ there exists $\alpha_{k} \in L^{1}(J)$ such that

$$
\sup _{\|x\|,|y| \leq k}|f(t, x, y)| \leq \alpha_{k}(t) \text { for a.a } t \in J .
$$

$\left(H_{8}\right)$ There exists a continuous function $p: J \rightarrow[0, \infty)$ such that

$$
|f(t, x, y)| \leq m(t) \Omega(\|x\|+|y|), \quad t \in J, \quad x \in C \text { and } y \in X,
$$

where $\Omega:[0, \infty) \rightarrow(0, \infty)$ is a continuous nondecreasing function and

$$
\int_{0}^{T} m(s) d s<\int_{c}^{\infty} \frac{d s}{s+\Omega(s)}<\infty
$$

where

$$
\begin{gathered}
m(t)=\max \left\{c_{1}\left[M c_{1}+M+M^{*}\right], M\left(c_{1} T+T+1\right) p(t)\right\}, \\
M=\sup \{|C(t)|: t \in J\}, \quad M^{*}=\sup \{|A S(t)|: t \in J\}, \\
c=\left(M+M^{*}+c_{1}\right)\|\phi\|+(1+T) M\left\{\left|y_{0}\right|+c_{1}\|\phi\|+c_{2}\right\}+\left(M+M^{*}\right) c_{2} T+c_{2} .
\end{gathered}
$$




\section{Main Result}

Theorem. Suppose $\left(H_{1}\right)-\left(H_{8}\right)$ hold. Then the IVP (1) has atleast one mild solution on $[-r, T]$.

Proof. Consider the space $Z=C([-r, T], X) \cap C^{1}(J, X)$ with the norm

$$
\|x\|^{*}=\max \left\{\|x\|_{r},\left\|x^{\prime}\right\|_{0}\right\},
$$

where

$$
\|x\|_{r}=\sup \{|x(t)|:-r \leq t \leq T\}, \quad\left\|x^{\prime}\right\|_{0}=\sup \left\{\left|x^{\prime}(t)\right|: 0 \leq t \leq T\right\} .
$$

To prove the existence of a mild solution of the IVP (1) we apply Lemma 4. First we obtain a priori bounds for the mild solutions of the IVP

$$
\frac{d}{d t}\left[x^{\prime}(t)-\lambda g\left(t, x_{t}\right)\right]=\lambda A x(t)+\lambda f\left(t, x_{t}, x^{\prime}(t)\right), \quad t \in J, \quad \lambda \in(0,1) .
$$

Let $x$ be a mild solution of the equation (2). From

$$
\begin{aligned}
x(t)= & \lambda C(t) \phi(0)+\lambda S(t)\left[y_{0}-g(0, \phi)\right]+\lambda \int_{0}^{t} C(t-s) g\left(s, x_{s}\right) d s \\
& +\lambda \int_{0}^{t} S(t-s) f\left(s, x_{s}, x^{\prime}(s)\right) d s, \quad t \in J,
\end{aligned}
$$

we have

$$
\begin{aligned}
|x(t)| \leq & M\|\phi\|+M T\left\{\left|y_{0}\right|+c_{1}\|\phi\|+2_{c_{2}}\right\}+M_{c_{1}} \int_{0}^{t}\left\|x_{s}\right\| d s \\
& +M T \int_{0}^{t} p(s) \Omega\left(\left\|x_{s}\right\|+\left|x^{\prime}(s)\right|\right) d s, \quad t \in J .
\end{aligned}
$$

Consider the function $\mu$ defined by

$$
\mu(t)=\sup \{|x(s)|:-r \leq s \leq t\}, \quad t \in J .
$$

Let $t^{*} \in[-r, t]$ be such that $\mu(t)=\left|x\left(t^{*}\right)\right|$. If $t^{*} \in[0, t]$, by the previous inequality we have,

$$
\begin{aligned}
\mu(t) \leq & M\|\phi\|+M T\left\{\left|y_{0}\right|+c_{1}\|\phi\|+2_{c_{2}}\right\}+M c_{1} \int_{0}^{t} \mu(s) d s \\
& +M T \int_{0}^{t} p(s) \Omega\left(\mu(s)+\left|x^{\prime}(s)\right|\right) d s \quad t \in J .
\end{aligned}
$$

If $t^{*} \in[-r, 0]$ then $\mu(t)=\|\phi\|$ and the previous inequality holds since $M \geq 1$. 
Denoting by $v(t)$ the right hand side of the above inequality we have,

$$
\mu(t) \leq v(t), \quad t \in J, v(0)=M\|\phi\|+M T\left\{\left|y_{0}\right|+c_{1}\|\phi\|+2_{c_{2}}\right\}
$$

and

$$
\begin{aligned}
v^{\prime}(t) & =M c_{1} \mu(t)+M T p(t) \Omega\left(\mu(t)+\left|x^{\prime}(t)\right|\right), \quad t \in J \\
& \leq M c_{1} v(t)+M T p(t) \Omega\left(v(t)+\left|x^{\prime}(t)\right|\right), \quad t \in J
\end{aligned}
$$

By

$$
\begin{aligned}
x^{\prime}(t)= & \lambda A S(t) \phi(0)+\lambda C(t)\left[y_{0}-g(0, \phi)\right]+\lambda g\left(t, x_{t}\right)+\lambda \int_{0}^{t} A S(t-s) g\left(s, x_{s}\right) d s \\
& +\lambda \int_{0}^{t} C(t-s) f\left(s, x_{s}, x^{\prime}(s)\right) d s, \quad t \in J
\end{aligned}
$$

we obtain

$$
\begin{aligned}
\left|x^{\prime}(t)\right| \leq & M^{*}\|\phi\|+M\left\{y_{0} \mid+c_{1}\|\phi\|+c_{2}\right\}+c_{1}\left\|x_{t}\right\|+c_{2}+M^{*}\left\{C_{2} T+c_{1} \int_{0}^{t}\left\|x_{s}\right\| d s\right\} \\
& +M \int_{0}^{t} p(s) \Omega\left(\left\|x_{s}\right\|+\left|x^{\prime}(s)\right|\right) d s, \quad t \in J .
\end{aligned}
$$

Denoting by $r(t)$ the right hand side of the above inequality we have

$$
\begin{aligned}
\left|x^{\prime}(t)\right| & \leq r(t), \quad t \in J, \\
r(0) & =M^{*}\|\phi\|+M\left\{\left|y_{0}\right|+c_{1}\|\phi\|+c_{2}\right\}+c_{1}\|\phi\|+c_{2}+M^{*} c_{2} T,
\end{aligned}
$$

and

$$
\begin{aligned}
r^{\prime}(t) & \leq c_{1} v^{\prime}(t)+M^{*} c_{1} v(t)+M p(t) \Omega(v(t)+r(t)), \quad t \in J, \\
& \leq c_{1}\left\{M c_{1} v(t)+M T p(t) \Omega(v(t)+r(t))\right\}+M^{*} c_{1} v(t)+M p(t) \Omega(v(t)+r(t)), \quad t \in J .
\end{aligned}
$$

Let

$$
w(t)=v(t)+r(t), \quad t \in J
$$

Then

$$
w(0)=c
$$

and

$$
\begin{aligned}
w^{\prime}(t) & =v^{\prime}(t)+r^{\prime}(t) \\
& \leq c_{1}\left[M c_{1}+M+M^{*}\right] v(t)+M\left(c_{1} T+T+1\right) p(t) \Omega(v(t)+r(t)) \\
& \leq c_{1}\left[M c_{1}+M+M^{*}\right] w(t)+M\left(c_{1} T+T+1\right) p(t) \Omega(w(t)) \\
& \leq m(t) w(t)+m(t) \Omega(w(t)), \quad t \in J
\end{aligned}
$$


This implies

$$
\int_{w(0)}^{w(t)} \frac{d s}{s+\Omega(s)} \leq \int_{0}^{T} m(s) d s<\int_{c}^{\infty} \frac{d s}{s+\Omega(s)}, \quad t \in J .
$$

This inequality implies that there is constant $K$ such that

$$
w(t)=v(t)+r(t) \leq K, \quad t \in J
$$

Then

$$
\begin{aligned}
|x(t)| & \leq v(t) \leq K, \quad t \in J \\
\left|x^{\prime}(t)\right| & \leq r(t) \leq K, \quad t \in J
\end{aligned}
$$

and hence

$$
\|x\|^{*}=\max \left\{\|x\|_{r},\left\|x^{\prime}\right\|_{0}\right\} \leq K,
$$

where $K$ depends only on $T$ and on the functions $m$ and $\Omega$.

We shall now prove that the operator $F: Z \rightarrow Z$ defined by

$$
\begin{aligned}
(F x)(t)= & \phi(t), \quad-r \leq t \leq 0, \\
(F x)(t)= & C(t) \phi(0)+S(t)\left[y_{0}-g(0, \phi)\right]+\int_{0}^{t} C(t-s) g\left(s, x_{s}\right) d s \\
& +\int_{0}^{t} S(t-s) f\left(s, x_{s}, x^{\prime}(s)\right) d s, \quad t \in J,
\end{aligned}
$$

is a completely continuous operator.

Let $B_{k}=\left\{x \in Z:\|x\|^{*} \leq k\right\}$ for some $k \geq 1$. We first show that $F$ maps $B_{k}$ into an equicontinuous family. Let $x \in B_{k}$ and $t_{1}, t_{2} \in J$. Then if $0<t_{1}<t_{2} \leq T$,

$$
\begin{aligned}
& \left|(F x)\left(t_{1}\right)-(F x)\left(t_{2}\right)\right| \\
\leq & \left|\left[C\left(t_{1}\right)-C\left(t_{2}\right)\right] \phi(0)\right|+\left|\left[S\left(t_{1}\right)-S\left(t_{2}\right)\right]\left[y_{0}-g(0, \phi)\right]\right| \\
& +\left|\int_{0}^{t_{1}}\left[C\left(t_{1}-s\right)-C\left(t_{2}-s\right)\right] g\left(s, x_{s}\right) d s\right|+\left|\int_{t_{1}}^{t_{2}} C\left(t_{2}-s\right) g\left(s, x_{s}\right) d s\right| \\
& +\left|\int_{0}^{t_{1}}\left[S\left(t_{1}-s\right)-S\left(t_{2}-s\right)\right] f\left(s, x_{s}, x^{\prime}(s)\right) d s\right| \\
& +\left|\int_{t_{1}}^{t_{2}} S\left(t_{2}-s\right) f\left(s, x_{s}, x^{\prime}(s)\right) d s\right| \\
\leq & \left|C\left(t_{1}\right)-C\left(t_{2}\right)\right|\|\phi\|+\left|S\left(t_{1}\right)-S\left(t_{2}\right)\right|\left\{\left|y_{0}\right|+c_{1}\|\phi\|+c_{2}\right\} \\
& +\int_{0}^{t_{1}}\left|C\left(t_{1}-s\right)-C\left(t_{2}-s\right)\right|\left\{c_{1}\left\|x_{s}\right\|+c_{2}\right\} d s \\
& +\int_{t_{1}}^{t_{2}}\left|C\left(t_{2}-s\right)\right|\left\{c_{1}\left\|x_{s}\right\|+c_{2}\right\} d s
\end{aligned}
$$




$$
\begin{aligned}
& +\int_{0}^{t_{1}}\left|S\left(t_{1}-s\right)-S\left(t_{2}-s\right)\right| \alpha_{k}(s) d s \\
& +\int_{t_{1}}^{t_{2}}\left|S\left(t_{2}-s\right)\right| \alpha_{k}(s) d s
\end{aligned}
$$

and similarly,

$$
\begin{aligned}
& \left|(F x)^{\prime}\left(t_{1}\right)-(F x)^{\prime}\left(t_{2}\right)\right| \\
\leq & \left|A\left(S\left(t_{1}\right)-S\left(t_{2}\right)\right) \phi(0)\right|+\left|\left[C\left(t_{1}\right)-C\left(t_{2}\right)\right]\left[y_{0}-g(0, \phi)\right]\right| \\
& +\left|g\left(t_{1}, x_{t_{1}}\right)-g\left(t_{2}, x_{t_{2}}\right)\right|+\left|\int_{0}^{t_{1}} A\left(S\left(t_{1}-s\right)-S\left(t_{2}-s\right)\right) g\left(s, x_{s}\right) d s\right| \\
& +\left|\int_{t_{1}}^{t_{2}} A S\left(t_{2}-s\right) g\left(s, x_{s}\right) d s\right| \\
& +\left|\int_{0}^{t_{1}}\left[C\left(t_{1}-s\right)-C\left(t_{2}-s\right)\right] f\left(s, x_{s}, x^{\prime}(s)\right) d s\right| \\
& +\left|\int_{t_{1}}^{t_{2}} C\left(t_{2}-s\right) f\left(s, x_{s}, x^{\prime}(s)\right) d s\right| \\
\leq & \left|A\left(S\left(t_{1}\right)-S\left(t_{2}\right)\right)\right||| \phi||+\left|\left[C\left(t_{1}\right)-C\left(t_{2}\right)\right]\right|\left\{\left|y_{0}\right|+c_{1}\|\phi\|+c_{2}\right\} \\
& +\left|g\left(t_{1}, x_{t_{1}}\right)-g\left(t_{2}, x_{t_{2}}\right)\right|+\int_{0}^{t_{1}}\left|A\left(S\left(t_{1}-s\right)-S\left(t_{2}-s\right)\right)\right|\left\{c_{1}\left\|x_{s}\right\|+c_{2}\right\} d s \\
& +\int_{t_{1}}^{t_{2}}\left|A S\left(t_{2}-s\right)\right|\left\{c_{1}|| x_{s} \|+c_{2}\right\} d s \\
& +\int_{0}^{t_{1}}\left|C\left(t_{1}-s\right)-C\left(t_{2}-s\right)\right| \alpha_{k}(s) d s \\
& +\int_{t_{1}}^{t_{2}}\left|C\left(t_{2}-s\right)\right| \alpha_{k}(s) d s,
\end{aligned}
$$

The right-hand side of (4) and (5) are independent of $y \in B_{k}$ and tends to zero as $t_{2}-t_{1} \rightarrow 0$, since $C(t), S(t)$ are uniformly continuous for $t \in J$ and the compactness of $C(t), S(t)$ for $t>0$ imply the continuity in the uniform operator topology. The compactness of $S(t)$ follows from that of $C(t)$ (see [9].)

Thus $F$ maps $B_{k}$ into an equicontinuous family of functions. It is easy to see that the family $F B_{k}$ is uniformly bounded.

Next we show $\overline{F B_{k}}$ is compact. Since we have shown $F B_{k}$ is an equicontinuous collection, it suffices by the Arzela-Ascoli theorem to show that $F$ maps $B_{k}$ into a precompact set in $X$.

Let $0<t \leq T$ be fixed and $\epsilon$ a real number satisfying $0<\epsilon<t$. For $x \in B_{k}$ we define

$$
\begin{aligned}
\left(F_{\epsilon} x\right)(t)= & C(t) \phi(0)+S(t)\left[y_{0}-g(0, \phi]+\int_{0}^{t-\epsilon} C(t-s) g\left(s, x_{s}\right) d s\right. \\
& +\int_{0}^{t-\epsilon} S(t-s) f\left(s, x_{s}, x^{\prime}(s)\right) d s, \quad t \in J
\end{aligned}
$$


Since $C(t), S(t)$ are compact operators, the set $Y_{\epsilon}(t)=\left\{\left(F_{\epsilon} x\right)(t): x \in B_{k}\right\}$ is precompact in $X$ for every $\epsilon, 0<\epsilon<t$. Moreover for every $x \in B_{k}$ we have

$$
\begin{aligned}
\left|(F x)(t)-\left(F_{\epsilon} x\right)(t)\right| \leq & \int_{t-\epsilon}^{t}\left|C(t-s) g\left(s, x_{s}\right)\right| d s \\
& +\int_{t-\epsilon}^{t}\left|S(t-s) f\left(s, x_{s}, x^{\prime}(s)\right)\right| d s \\
\leq & \int_{t-\epsilon}^{t}|C(t-s)|\left\{c_{1}|| x_{s}||+c_{2}\right\} d s \\
& \int_{t-\epsilon}^{t}|S(t-s)| \alpha_{k}(s) d s,
\end{aligned}
$$

and

$$
\begin{aligned}
& \left|(F x)^{\prime}(t)-\left(F_{\epsilon} x\right)^{\prime}(t)\right| \\
\leq & \left|g\left(t, x_{t}\right)-C(\epsilon) g\left(t-\epsilon, x_{t-\epsilon}\right)\right|+\int_{t-\epsilon}^{t}\left|A S(t-s) g\left(s, x_{s}\right)\right| d s \\
& +\int_{t-\epsilon}^{t}\left|C(t-s) f\left(s, x_{s}, x^{\prime}(s)\right)\right| d s \\
\leq & \left|g\left(t, x_{t}\right)-C(\epsilon) g\left(t-\epsilon, x_{t-\epsilon}\right)\right|+\int_{t-\epsilon}^{t}|A S(t-s)|\left\{c_{1}\left\|x_{s}\right\|+c_{2}\right\} d s \\
& +\int_{t-\epsilon}^{t}|C(t-s)| \alpha_{k}(s) d s,
\end{aligned}
$$

Therefore there are precompact sets arbitrarily close to the set $\left\{(F x)(t): x \in B_{k}\right\}$. Hence the set $\left\{(F x)(t): x \in B_{k}\right\}$ is precompact in $X$.

It remains to show that $F: Z \rightarrow Z$ is continuous. Let $\left\{x_{n}\right\}_{0}^{\infty} \subseteq Z$ with $x_{n} \rightarrow x$ in $Z$. Then there is an integer $q$ such that $\left|x_{n}(t)\right| \leq q,\left|x_{n}^{\prime}(t)\right| \leq q$ for all $n$ and $t \in J$, so $|x(t)| \leq q,\left|x^{\prime}(t)\right| \leq q$ and $x, x^{\prime} \in Z$. By $\left(H_{7}\right)$

$$
f\left(t, x_{n t}, x_{n}{ }^{\prime}(t)\right) \rightarrow f\left(t, x_{t}, x^{\prime}(t)\right)
$$

for each $t \in J$ and since

$$
\left|f\left(t, x_{n t}, x_{n}{ }^{\prime}(t)\right)-f\left(t, x_{t}, x^{\prime}(t)\right)\right| \leq 2 \alpha_{q}(t),
$$

we have by dominated convergence theorem,

$$
\begin{aligned}
\left\|F x_{n}-F x\right\|= & \sup _{t \in J} \mid \int_{0}^{t} C(t-s)\left[g\left(s, x_{n s}\right)-g\left(s, x_{s}\right)\right] d s \\
& +\int_{0}^{t} S(t-s)\left[f\left(s, x_{n s}, x_{n}{ }^{\prime}(s)\right)-f\left(s, x_{s}, x^{\prime}(s)\right)\right] d s \mid
\end{aligned}
$$




$$
\begin{aligned}
\leq & \int_{0}^{T}\left|C(t-s)\left[g\left(s, x_{n s}\right)-g\left(s, x_{s}\right)\right]\right| d s \\
& +\int_{0}^{T}\left|S(t-s)\left[f\left(s, x_{n s}, x_{n}{ }^{\prime}(s)\right)-f\left(s, x_{s}, x^{\prime}(s)\right)\right]\right| d s \rightarrow 0 \quad \text { as } n \rightarrow \infty
\end{aligned}
$$

and

$$
\begin{aligned}
& \left\|\left(F x_{n}\right)^{\prime}-(F x)^{\prime}\right\| \\
= & \sup _{t \in J} \mid\left[g\left(t, x_{n t}\right)-g\left(t, x_{t}\right)\right]+\int_{0}^{t} A S(t-s)\left[g\left(s, x_{n s}\right)-g\left(s, x_{s}\right)\right] d s \\
& +\int_{0}^{t} C(t-s)\left[f\left(s, x_{n s}, x_{n}{ }^{\prime}(s)\right)-f\left(s, x_{s}, x^{\prime}(s)\right)\right] d s \mid \\
\leq & \left|g\left(t, x_{n t}\right)-g\left(t, x_{t}\right)\right| d s+\int_{0}^{t}\left|A S(t-s)\left[g\left(s, x_{n s}\right)-g\left(s, x_{s}\right)\right]\right| d s \\
& +\int_{0}^{T}\left|C(t-s)\left[f\left(s, x_{n s}, x_{n}{ }^{\prime}(s)\right)-f\left(s, x_{s}, x^{\prime}(s)\right)\right] d s\right| r \rightarrow 0, \text { as } n \rightarrow \infty .
\end{aligned}
$$

Thus $F$ is continuous. This completes the proof that $F$ is completely continuous.

Obviously, the set $\xi(F)=\{x \in Z: x=\lambda F x, \lambda \in(0,1)\}$ is bounded, as we proved in the first step. Consequently by Schaefer's theorem the operator $F$ has a fixed point in $Z$. This means that any fixed point of $F$ is a mild solution of $(1)$ on $[-r, T]$ satisfying $(F x)(t)=x(t)$. Thus IVP $(1)$ has atleast one mild solution on $[-r, T]$.

\section{Example}

Consider the following partial differential equation

$$
\begin{aligned}
\frac{\partial}{\partial t}\left(z_{t}(y, t)-\mu(t, z(y, t-r)\right. & =z_{y y}(y, t)+\int_{0}^{t} \sigma\left(s, z(y, s-r), z_{s}(y, s)\right) d s, \\
z(0, t) & =z(\pi, t)=0, \quad \text { for } t>0, \\
z(y, t) & =\phi(y, t), \quad \text { for }-r \leq t \leq 0, \\
z_{t}(y, 0) & =z_{1}(y), \quad t \in J=[0, T], \text { for } 0<y<\pi,
\end{aligned}
$$

where $\phi$ is continuous.

Let $X=L^{2}[0, \pi]$ and let $A: X \rightarrow X$ be defined by

$$
A w=w^{\prime \prime}, \quad w \in D(A),
$$

where $D(A)=\left\{w \in X: w, w^{\prime}\right.$ are absolutely continuous, $\left.w^{\prime \prime} \in X, w(0)=w(\pi)=0\right\}$. Then,

$$
A w=\sum_{n=1}^{\infty}-n^{2}\left(w, w_{n}\right) w_{n}, \quad w \in D(A)
$$


where $w_{n}(s)=\sqrt{2 / \pi} \sin n s, n=1,2,3, \ldots$ is the orthogonal set of eigenvalues of $A$.

It can be easily shown that $A$ is the infinitesimal generator of a strongly continuous cosine family $C(t), t \in R$, in $X$ and is given by

$$
C(t) w=\sum_{n=1}^{\infty} \cos n t\left(w, w_{n}\right) w_{n}, \quad w \in X .
$$

The associated sine family is given by

$$
S(t) w=\sum_{n=1}^{\infty} \frac{1}{n} \sin n t\left(w, w_{n}\right) w_{n}, \quad w \in X .
$$

Let $g: J \times C \rightarrow X$ be defined by

$$
g(t, u)(y)=\mu(t, u(y)), \quad u \in C, \quad y \in[0, \pi]
$$

where $\mu: J \times[0, \pi] \rightarrow[0, \pi]$ is continuous and strongly measurable. Also there exist positive constants $c_{1}$ and $c_{2}$ such that

$$
\| \mu\left(t, \phi\left\|\leq c_{1}\right\| \phi \|+c_{2} .\right.
$$

Let $f: J \times C \times X \rightarrow X$ be defined by

$$
f(t, v, w)(y)=\sigma(t, v(y), w(y)), \quad v \in C, w \in X, \quad y \in[0, \pi],
$$

where $\sigma: J \times[0, \pi] \times[0, \pi] \rightarrow[0, \pi]$ is continuous and strongly measurable. Further the function $\sigma$ satisfies the following condition:

There exists a continuous function $p: J \rightarrow[0, \infty)$ such that

$$
\|\sigma(t, v, w)\| \leq p(t) \Omega(\|v\|+|w|), \quad t \in J, v \in C, w \in X
$$

whrere $\Omega:[0, \infty) \rightarrow(0, \infty)$ is a continuous nondecreasing function such that

$$
\int_{0}^{T} p(s) d s<\int_{c}^{\infty} \frac{d s}{s+\Omega(s)}
$$

and $c$ is a known constant.

With this choice of $A, g$ and $f,(1)$ is an abstract formulation of (6). Furthermore, all the conditions stated in the above theorem are statisfied. hence the equation (6) has atleast one mild solution on $[-r, T]$.

\section{References}

[1] J. Ball, Initial boundary value problems for an extensible beam, Journal of Mathematical Analysis and Applications 42(1973), 61-90. 
[2] J. Bochenek, An abstract nonlinear second order differential equation, Annales Polonici Mathematici 54(1991), 155-166.

[3] W. E. Fitzgibbon, Global existence and boundedness of solutions to the extensiblc beam equation, SIAM Journal of Mathematical Analysis 13(1982), 739-745.

[4] E. Hernández and H. R. Henríquez, Existence results for partial neutral functional integrodifferential equations with unbounded delay, Journal of mathematical Analysis and Applications 221(1998), 452-475.

[5] S. K. Ntouyas, Global existence for neutral functional integrodifferential equations, Nonlinear Analysis: Theory, Methods and Applications 30(1997), 2133-2142.

[6] S. K. Ntouyas and P. Ch. Tsamatos, Global existence for second order semilinear ordinary and delay integrodifferential equations with nonlocal conditons, Applicable Analysis, 67(1997), 245-257.

[7] H. Schaefer, Uber die methode der a priori schranken, Mathematische Annalem 129(1955), 415-416.

[8] C. C. Travis and G. F. Webb, Cosine families and abstract nonlinear second order differential equations, Acta Mathematica Academiae Scientiarum Hungaricae 32(1978), 75-96.

[9] C. C. Travis and G. F. Webb, Compactness, regularity and uniform continuity properties of strongly continuous cosine families, Houston Journal of Mathematics 3(1977), 555-567.

Department of Mathematics, Bharathiar University, Coimbatore -641 046, INDIA. 